\title{
Measurement of $\alpha_{s}$ from the Structure of Particle Clusters Produced in Hadronic $Z$ Decays
}

\author{
The ALEPH Collaboration
}

\begin{abstract}
Using 106000 hadronic events obtained with the ALEPH detector at LEP at energies close to the $Z$ resonance peak, the strong coupling constant $\alpha_{2}$ is measured by an analysis of energy-energy correlations (EEC) and the global event shape variables Thrust, C-parameter and Oblateness. It is shown that the theoretical uncertainties can be significantly reduced if the final state particles are first combined in clusters using a minimum scaled invariant mass cut, $y_{c u t}$, before these variables are computed. The combined result from all shape variables of pre-clustered events is $\alpha_{s}\left(M_{Z}^{2}\right)=0.117 \pm 0.005$ for a renormalization scale $\mu=M_{Z} / 2$. For $\mu$ values between $M_{Z}$ and the b-quark mass, the result changes by ${ }_{-0.009}^{+0.006}$.
\end{abstract}




\section{The ALEPH Collaboration}

D. Decamp, B. Deschizeaux, C. Goy, J.-P. Lees, M.-N. Minard Laboratoire de Physique des Particules (LAPP), IN $P^{3}$-CNRS, 74019 Annecy-le-Vieux Cedex, France

R. Alemany, J.M. Crespo, M. Delfino, E. Fernandez, V. Gaitan, Ll. Garrido, P. Mato, R. Miquel, Ll.M. Mir, S. Orteu, A. Pacheco, J.A. Perlas, E. Tubau Laboratorio de Fisica de Altas Energias, Universidad Autonoma de Barcelona, 08193 Bellaterra (Barcelona), Spain ${ }^{9}$

M.G. Catanesi, D. Creanza, M. de Palma, A. Farilla, G. Iaselli, G. Maggi, M. Maggi, S. Natali, S. Nuzzo, M. Quattromini, A. Ranieri, G. Raso, F. Romano, F. Ruggieri, G. Selvaggi, L. Silvestris, P. Tempesta, G. Zito INFN Sezione di Bari e Dipartimento di Fisica dell' Università, 70126 Bari, Italy Y. Gao, H. Hu, ${ }^{22}$ D. Huang, X. Huang, J. Lin, J. Lou, C. Qiao, ${ }^{22}$ T. Ruan, ${ }^{22}$ Wang, Y. Xie, D. Xu, R. Xu, J. Zhang,
W. Zhao

Institute of High-Energy Physics, Academia Sinica, Beijing, The People's Republic of China ${ }^{10}$

H. Albrecht, W.B. Atwood, F. Bird, E. Blucher, G. Bonvicini, F. Bossi, D. Brown, T.H. Burnett, H. Drevermann,

F. Dydak, R.W. Forty, C. Grab, R. Hagelberg, S. Haywood, B. Jost, M. Kasemann, G. Kellner, J. Knobloch,

A. Lacourt, I. Lehraus, T. Lohse, D. Lüke, A. Marchioro, M. Martinez, J. May, S. Menary, A. Minten, A. Miotto,

J. Nash, P. Palazzi, F. Ranjard, G. Redlinger, A. Roth, J. Rothberg, H. Rotscheidt, W. von Rüden, R. St.Denis,

D. Schlatter, M. Takashima, M. Talby, W. Tejessy, H. Wachsmuth, S. Wasserbaech, S. Wheeler, W. Wiedenmann,

W. Witzeling, J. Wotschack

European Laboratory for Particle Physics (CERN), 1211 Geneva 23, Switzerland

Z. Ajaltouni, M. Bardadin-Otwinowska, A. Falvard, R. El Fellous, P. Gay, J. Harvey, P. Henrard, J. Jousset,

B. Michel, J-C. Montret, D. Pallin, P. Perret, J. Proriol, F. Prulhière, G. Stimpfl

Laboratoire de Physique Corpusculaire, Université Blaise Pascal, IN ${ }^{2} P^{3}-C N R S$, Clermont-Ferrand, 63177 Aubière, France

J.D. Hansen, J.R. Hansen, P.H. Hansen, R. Møllerud, E.R. Nielsen, B.S. Nilsson

Niels Bohr Institute, 2100 Copenhagen, Denmark ${ }^{11}$

I. Efthymiopoulos, E. Simopoulou, A. Vayaki

Nuclear Research Center Demokritos (NRCD), Athens, Greece

J. Badier, A. Blondel, G. Bonneaud, J. Bourotte, F. Braems, J.C. Brient, G. Fouque, A. Gamess, R. Guirlet,

A. Rosowsky, A. Rougé, M. Rumpf, R. Tanaka, H. Videau

Laboratoire de Physique Nucléaire et des Hautes Energies, Ecole Polytechnique, IN ${ }^{2} P^{3}{ }_{-C N R S}, 91128$

Palaiseau Cedex, France

D.J. Candlin, E. Veitch

Department of Physics, University of Edinburgh, Edinburgh EH9 3JZ, United Kingdom ${ }^{12}$

G. Parrini

Dipartimento di Fisica, Università di Firenze, INFN Sezione di Firenze, 50125 Firenze, Italy

M. Corden, C. Georgiopoulos, M. Ikeda, J. Lannutti, D. Levinthal ${ }^{17}$ M. Mermikides, L. Sawyer

Supercomputer Computations Research Institute and Dept. of Physics, Florida State University,

Tallahassee, FL 32306, USA ${ }^{14,15,16}$

A. Antonelli, R. Baldini, G. Bencivenni, G. Bologna, P. Campana, G. Capon, V. Chiarella, B. D'Ettorre-Piazzoli?

G. Felici, P. Laurelli, G. Mannocchi?, F. Massimo-Brancaccio, F. Murtas, G.P. Murtas, G. Nicoletti, L. Passalacqua,

M. Pepe-Altarelli, P. Picchi, P. Zografou

Laboratori Nazionali dell'INFN (LNF-INFN), 00044 Frascati, Italy 
B. Altoon, O. Boyle, A.W. Halley, I. ten Have, J.L. Hearns, J.G. Lynch, W.T. Morton, C. Raine, J.M. Scarr, K. Smith, A.S. Thompson, R.M. Turnbull Department of Physics and Astronomy, University of Glasgow, Glasgow G12 8QQ, United Kingdom ${ }^{12}$

B. Brandl, O. Braun, R. Geiges, C. Geweniger, P. Hanke, V. Hepp, E.E. Kluge, Y. Maumary, A. Putzer, B. Rensch, A. Stahl, K. Tittel, M. Wunsch Institut für Hochenergiephysik, Universität Heidelberg, 6900 Heidelberg, Fed. Rep. of Germany ${ }^{18}$

A.T. Belk, R. Beuselinck, D.M. Binnie, W. Cameron, M. Cattaneo, P.J. Dornan, S. Dugeay, A.M. Greene, J.F. Hassard, N.M. Lieske, S.J. Patton, D.G. Payne, M.J. Phillips, J.K. Sedgbeer, G. Taylor, I.R. Tomalin, A.G. Wright Department of Physics, Imperial College, London SW7 2BZ, United Kingdom ${ }^{12}$

P. Girtler, D. Kuhn, G. Rudolph Institut für Experimentalphysik, Universität Innsbruck, 6020 Innsbruck, Austria ${ }^{20}$

C.K. Bowdery, T.J. Brodbeck, A.J. Finch, F. Foster, G. Hughes, N.R. Keemer, M. Nuttall, A. Patel, B.S. Rowlingson, T. Sloan, S.W. Snow, E.P. Whelan

Department of Physics, University of Lancaster, Lancaster LA1 4YB, United Kingdom ${ }^{12}$

T. Barczewski, L.A.T. Bauerdick, K. Kleinknecht, B. Renk, S. Roehn, H.-G. Sander, M. Schmelling, H. Schmidt, F. Steeg Institut für Physik, Universität Mainz, 6500 Mainz, Fed. Rep. of Germany ${ }^{18}$

J-P. Albanese, J-J. Aubert, C. Benchouk, V. Bernard, A. Bonissent, D. Courvoisier, F. Etienne, S. Papalexiou, P. Payre, B. Pietrzyk, Z. Qian

Centre de Physique des Particules, Faculté des Sciences de Luminy, IN $P^{3}$-CNRS, 13288 Marseille, France

W. Blum, P. Cattaneo, G. Cowan, B. Dehning, H. Dietl, M. Fernandez-Bosman, T. Hansl-Kozanecka, ${ }^{23}$ A. Jahn, W. Kozanecki, ${ }^{3,24}$ E. Lange, G. Lütjens, G. Lutz, W. Männer, H-G. Moser, Y. Pan, R. Richter, J. Schrōder, A.S. Schwarz, R. Settles, U. Stierlin, J. Thomas, G. Wolf

Max-Planck-Institut für Physik und Astrophysik, Werner-Heisenberg-Institut für Physik, 8000 München, Fed. Rep. of Germany ${ }^{18}$

V. Bertin, G. de Bouard, J. Boucrot, O. Callot, X. Chen, A. Cordier, M. Davier, G. Ganis, J.-F. Grivaz, Ph. Heusse, P. Janot, V. Journé, D.W. Kim,' J. Lefrançois, A.-M. Lutz, J.-J. Veillet, I. Videau, Z. Zhang, F. Zomer Laboratoire de l'Accéléateur Linéaire, Université de Paris-Sud, IN $P^{3}$-CNRS, 91405 Orsay Cedex, France

S.R. Amendolia, G. Bagliesi, G. Batignani, L. Bosisio, U. Bottigli, C. Bradaschia, M. Carpinelli, M.A. Ciocci, R. Dell'Orso, I. Ferrante, F. Fidecaro, L. Foà, E. Focardi, F. Forti, A. Giassi, M.A. Giorgi, F. Ligabue, A. Lusiani, E.B. Mannelli, P.S. Marrocchesi, A. Messineo, L. Moneta, F. Palla, G. Sanguinetti, J. Steinberger, R. Tenchini, G. Tonelli, G. Triggiani, C. Vannini-Castaldi, A. Venturi, P.G. Verdini, J. Walsh Dipartimento di Fisica dell'Università, INFN Sezione di Pisa, e Scuola Normale Superiore, 56010 Pisa, Italy

J.M. Carter, M.G. Green, P.V. March, T. Medcalf, I.S. Quazi, M.R. Saich, J.A. Strong, R.M. Thomas, L.R. West, T. Wildish Department of Physics, Royal Holloway \& Bedford New College, University of London, Surrey TW20 OEX, United Kingdom ${ }^{12}$

D.R. Botterill, R.W. Clift, T.R. Edgecock, M. Edwards, S.M. Fisher, T.J. Jones, P.R. Norton, D.P. Salmon, J.C. Thompson Particle Physics Dept., Rutherford Appleton Laboratory, Chilton, Didcot, OXON OX11 OQX, United Kingdom ${ }^{12}$ 
B. Bloch-Devaux, P. Colas, C. Klopfenstein, E. Lançon, E. Locci, S. Loucatos, E. Monnier, P. Perez, F. Perrier, J. Randex, J.-F. Renardy, A. Roussarie, J.-P. Schuller, J. Schwindling Département de Physique des Particules Élémentaires, CEN-Saclay, 91191 Gif-sur-Yvette Cedex, France ${ }^{19}$

J.G. Ashman, C.N. Booth, C. Buttar, R. Carney, S. Cartwright, F. Combley, M. Dinsdale, M. Dogru, F. Hatfield,

J. Martin, D. Parker, P. Reeves, L.F. Thompson Department of Physics, University of Sheffield, Sheffield S3 7RH, United Kingdom 12

S. Brandt, H. Burkhardt, C. Grupen, H. Meinhard, L. Mirabito, E. Neugebauer, U. Schäfer, H. Seywerd Fachbereich Physik, Universität Siegen, 5900 Siegen, Fed. Rep. of Germany ${ }^{18}$

G. Apollinari, G. Giannini, B. Gobbo, F. Liello, L. Rolandi, U. Stiegler Dipartimento di Fisica, Università di Thieste e INFN Sezione di Trieste, 34127 Trieste, Italy

L. Bellantoni, J.F. Boudreau, D. Cinabro, J.S. Conway, D.F. Cowen, ${ }^{25}$ A.J. DeWeerd, Z. Feng, D.P.S. Ferguson, Y.S. Gao, J. Grahl, J.L. Harton, J. Hilgart, J.E. Jacobsen, R.C. Jared, R.P. Johnson, B.W. LeClaire, Y.B. Pan, J.R. Pater, Y. Saadi, V. Sharma, M.A. Walsh, J.A. Wear, F.V. Weber, M.H. Whitney, Sau Lan Wu, Z.L. Zhou, G. Zobernig

Department of Physics, University of Wisconsin, Madison, WI 53706, USA ${ }^{13}$

\footnotetext{
${ }^{1}$ Now at CERN.

${ }^{2}$ Permanent address: DESY, Hamburg, Fed. Rep. of Germany.

${ }^{3}$ On leave of absence from SLAC, Stanford, CA 94309, USA.

${ }^{4}$ On leave of absence from University of Washington, Seattle, WA 98195, USA.

${ }^{5}$ Also Centre de Physique des Particules, Faculté des Sciences, Marseille, France

${ }^{6}$ Also Istituto di Fisica Generale, Università di Torino, Torino, Italy.

${ }^{7}$ Also Istituto di Cosmo-Geofisica del C.N.R., Torino, Italy.

${ }^{8}$ Permanent address: LBL, Berkeley, CA 94720, USA.

'Supported by CAICYT, Spain.

${ }^{10}$ Supported by the National Science Foundation of China.

${ }^{11}$ Supported by the Danish Natural Science Research Council.

${ }^{12}$ Supported by the UK Science and Engineering Research Council.

${ }^{13}$ Supported by the US Department of Energy, contract DE-AC02-76ER00881.

${ }^{14}$ Supported by the US Department of Energy, contract DE-FG05-87ER40319.

${ }^{15}$ Supported by the NSF, contract PHY-8451274.

${ }^{16}$ Supported by the US Department of Energy, contract DE-FCOS-85ER250000.

${ }^{17}$ Supported by SLOAN fellowship, contract BR 2703.

${ }^{18}$ Supported by the Bundesministerium für Forschung und Technologie, Fed. Rep. of Germany.

${ }^{19}$ Supported by the Institut de Recherche Fondamentale du C.E.A..

${ }^{20}$ Supported by Fonds zur Förderung der wissenschaftlichen Forschung, Austria.

${ }^{21}$ Supported by the Korean Science and Engineering Foundation and Ministry of Education.

${ }^{22}$ Supported by the World Laboratory.

${ }^{23} \mathrm{On}$ leave of absence from MIT, Cambridge, MA 02139, USA.

${ }^{24}$ Supported by Alexander von Humboldt Fellowship, Germany.

${ }^{25}$ Now at California Institute of Technology, Pasadena, California, USA.
} 


\section{Introduction}

The determination of the strong coupling constant $\alpha_{,}\left(M_{Z}^{2}\right)$ from the structure of hadronic events at LEP energies generally requires direct comparison of data with the structure of partonic final states as calculated in second order perturbative Quantum Chromo-Dynamics (QCD). The event shape is characterized by a set of variables which are stable under soft or collinear gluon emission, sensitive to three jet topologies but not sensitive to larger jet multiplicities and to the hadronization process. The most successful global event shape variable, the differential two jet rate, has lead to consistent measurements of $\alpha_{0}$ by MARK II [1] and the LEP experiments $[2,3]$. Other variables, like thrust, oblateness, C-parameter, the larger invariant mass, and the invariant mass difference of the two thrust hemispheres, are still strongly affected by higher order QCD corrections and/or the hadronization of partons, and are thus less useful for a precise determination of $\alpha_{0}[3]$.

This paper shows that the theoretical uncertainties can be reduced by considering variables which are not computed for the single particle momenta of the final state (as done in our earlier work [3]) but for clusters of neighbouring particles in phase space. Naively, these clusters should more closely resemble the structure of a purely partonic final state as accessible in finite order perturbation theory.

The paper is organized as follows: after a description of the event selection and data correction in section 2 , the analysis strategies are discussed in section 3 . The analysis of energy-energy correlations and the influence of pre-clustering are presented in section 4 . In section 5 these new methods are applied for global event shape variables. In section 6 the results from all event shape variables are combined.

\section{Event Selection and Data Correction}

The detector, which provides both tracking information and calorimetry over almost the full solid angle, is described in detail in reference [4].

The trigger required at least $6.5 \mathrm{GeV}$ in the barrel of the electromagnetic calorimeter or $3.8 \mathrm{GeV}$ in either endcap or $1.6 \mathrm{GeV}$ in both endcaps. A second, penetrating particle trigger required hits in at least four planes of the hadron calorimeter in the same azimuthal region as at least 6 coordinates in the Inner Tracking Chamber (ITC). The combined efficiency of the two triggers was $100 \%$ for hadronic events.

The analysis is based on 106000 hadronic events at center-of-mass energies in the range $91.0 \mathrm{GeV} \leq E_{C M} \leq 91.5 \mathrm{GeV}$. Only charged particles measured in the ITC and the Time Projection Chamber (TPC) of the ALEPH detector are used. The tracks are required to have at least four three-dimensional coordinates from the TPC and to extrapolate to within $2 \mathrm{~cm}$ of the beam line and to within $5 \mathrm{~cm}$ of the origin in the direction along the beam. In addition, the angle with respect to the beam axis is required to be at least $20^{\circ}$, and the momentum component in the direction perpendicular to the beam must be at least $200 \mathrm{MeV} / \mathrm{c}$. Using tracks which meet these criteria, the sphericity axis and the total charged energy are computed. Events are required to have at least five accepted charged tracks, the polar angle of the sphericity axis between $35^{\circ}$ and $145^{\circ}$, and a total charged energy of at least $15 \mathrm{GeV}$.

The experimental distributions are constructed using only charged particle information. They are corrected for the effects of geometrical acceptance, detector efficiency and resolution, decays, missing neutrals, secondary interactions and initial state photon radiation, using hadronic event generators and the detector simulation program. The systematic experimental 
errors are estimated from the uncertainties in these corrections. Details of the procedure can be found in references $[3,5]$.

\section{Analysis}

\subsection{Definition of Clusters}

Before computing event shape variables the final state particles are combined in clusters, with every particle initially representing a cluster. For each pair $(i, j)$ of clusters a scaled mass $y_{i j}$ is defined according to the JADE metric [6]

$$
y_{i j}=\frac{2 E_{i} E_{j}}{E_{v i s}^{2}} \cdot\left(1-\cos \Theta_{i j}\right)
$$

where $\Theta_{i j}$ is the angle between the momentum vectors $\vec{p}_{i}, \vec{p}_{j}$ of the clusters, $E_{i}, E_{j}$ are their energies, and $E_{v i}$ is the total visible energy in the event. If the pair with the smallest $y_{i j}$ fulfills $y_{i j}<y_{c u t}$, the corresponding clusters are combined according to the so called $\mathrm{E}_{0}$ recombination scheme [8]

$$
\begin{aligned}
E_{i j} & =E_{i}+E_{j} \\
\vec{p}_{i j} & =\left(\vec{p}_{i}+\vec{p}_{j}\right) \cdot \frac{E_{i}+E_{j}}{\left|\vec{p}_{i}+\vec{p}_{j}\right|}
\end{aligned}
$$

and the procedure is repeated. The $\mathrm{E}_{0}$ scheme was preferred over alternative schemes [8] because it was found to have consistently the smallest sensitivity to fragmentation processes.

\subsection{Second Order QCD Predictions}

As the basic theoretical ingredient the second order QCD matrix elements as calculated by Ellis, Ross and Terrano (ERT) [7] are used. These can be integrated to predict the distribution for a given event shape variable, $X$, as described for example in reference [8],

$$
\frac{1}{\sigma_{0}} \frac{\mathrm{d} \sigma}{\mathrm{d} X}=\frac{\alpha_{s}\left(\mu^{2}\right)}{2 \pi} A(X)+\left(\frac{\alpha_{s}\left(\mu^{2}\right)}{2 \pi}\right)^{2}\left[A(X) \cdot 2 \pi b_{0} \log \left(\frac{\mu^{2}}{s}\right)+B(X)\right]+\mathcal{O}\left(\alpha_{s}^{3}\right)
$$

with

$$
b_{0}=\frac{33-2 n_{f}}{12 \pi} .
$$

Here, $n_{f}(=5)$ denotes the number of active flavours and $\sqrt{s}$ is the center-of-mass energy. The functions $A$ and $B$ are specific to the particular event shape variable and contain the full information of the second order matrix elements. The parameter $\mu$ denotes the renormalization scale used for the calculation. Equation (1) is invariant under changes of $\mu$ up to $\mathcal{O}\left(\alpha_{a}^{2}\right)$, i.e. up to the finite order in which the perturbative calculation is done. The values of $A$ and $B$ for most of the classical event shape variables are tabulated in reference [8] for the $\overline{\mathrm{MS}}$ renormalization scheme.

In the case of shape variables for pre-clustered events, the functions $A$ and $B$ in equation (1) also depend on the value chosen for $y_{\text {cut }}$ and had to be specially computed for this analysis. This was done following the prescriptions in reference [8]. A numerical integration program [9], based on importance sampling and event weighting techniques, was used to generate partonic final states according to the ERT matrix elements. However, instead of calculating event shape variables for these partons, the program was modified such that the same clustering mechanism is applied on the parton level as in the analysis of the real data. An example of the result is 
shown in figure 1 which displays the functions $A\left(\cos \chi, y_{\text {cut }}\right), B\left(\cos \chi, y_{\text {cut }}\right)$ for $\sin ^{2} \chi \cdot \mathrm{EEC}(\cos \chi)$, where $\operatorname{EEC}(\chi)$ is the energy-energy correlation (defined below), at several values of $y_{\text {cut }}$. As can be seen, second order QCD predicts a specific dependence of the distribution on $y_{\text {cut }}$ which can be confronted with the data.

EEC in second order QCD
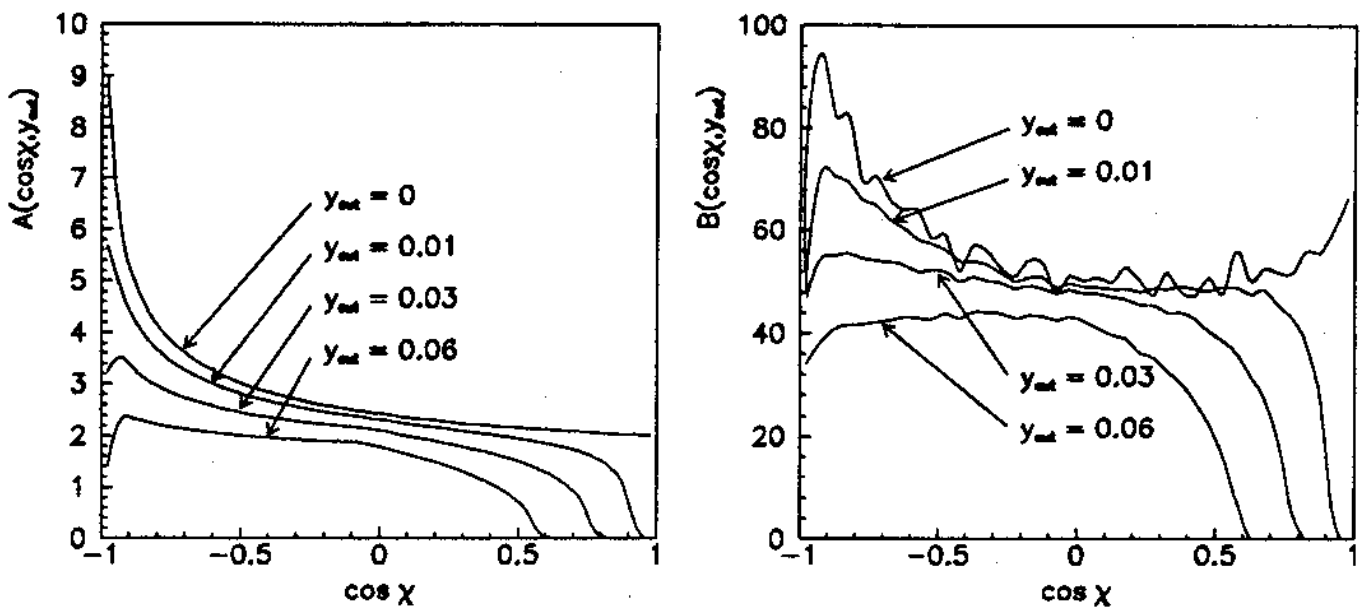

Figure 1: Second order $Q C D$ prediction for the functions $A, B$ of the $E E C$ after pre-clustering(see text). The fluctuations are due to the finite statistics of the Monte Carlo integration.

\subsection{Choice of Renormalization Scale}

Comparing equation (1) to the data one can now determine $\alpha_{s}\left(\mu^{2}\right)$ for any choice of the renormalization scale $\mu$ and then translate it into $\alpha_{s}\left(M_{Z}^{2}\right)$ using the two-loop expression for the running coupling constant [8]

$$
\alpha_{s}\left(\mu^{2}\right)=\frac{1}{b_{0} \log \left(\mu^{2} / \Lambda^{2}\right)}\left(1-\frac{b_{1} \log \left(\log \left(\mu^{2} / \Lambda^{2}\right)\right)}{b_{0}^{2} \log \left(\mu^{2} / \Lambda^{2}\right)}\right)
$$

with

$$
b_{1}=\frac{153-19 n_{f}}{24 \pi^{2}} .
$$

This results in a unique value for $\alpha_{s}\left(M_{Z}^{2}\right)$ to order $\alpha_{s}^{2}$. The choice of $\mu$ does, however, enter the result to third order, reflecting our ignorance of higher order corrections. Since $\alpha_{\mathrm{g}}$ is relatively large this third order effect still has a significant impact on the numerical results and constitutes a source of theoretical uncertainty.

There exist several theoretical prescriptions to reduce the sensitivity to the scale by choosing appropriate and typically very small values for $\mu[10]$. Since none of these methods can guarantee that third order terms become small, however, we prefer to determine the value $\alpha_{s}\left(M_{Z}^{2}\right)$ using $\mu=M_{Z}$ and to give an explicit parameterization of the shifts induced if the scale is varied. This allows to compare the results to other experiments with different choices of $\mu$.

In this paper the impact of higher order contributions is estimated by means of parton shower Monte Carlo models based on the leading-logarithm approximation, as described in the next section. In addition the renormalization scale is allowed to vary between $M_{Z}$ (which is taken to be $91.2 \mathrm{GeV}$ ) and the b-quark mass. Like in reference [3], the results, originally obtained at $\mu=M_{Z}$, are translated to an intermediate scale of $\mu=M_{Z} / 2$ using the parameterizations of the scale dependence. 


\subsection{Correction for Fragmentation and Systematic Errors}

The methods used to extract $\alpha_{s}\left(M_{Z}^{2}\right)$ from distributions of event shape variables and to estimate the theoretical uncertainties resemble closely those described in reference [3], where more details can be found. The fit results for $\alpha_{s}\left(M_{Z}^{2}\right)$ are corrected for the effects of higher order perturbative effects and for the effects of hadronization (both together in the following being referred to as effects of fragmentation) using the Lund second order matrix element (ME) model (JETSET 7.2) [11]. This model is based on the ERT matrix elements, which were also used for the theoretical predictions, and applies a scale $\mu^{2}=s, \sqrt{s}$ being the center-of-mass energy ( $\sqrt{s}=M_{Z}$ in this analysis). The model parameters were tuned to the data. For all event shape distributions under consideration the model reproduces the data well in the intervals used for the determination of $\alpha_{a}$. The correction was performed using the following procedure:

- For any global event shape variable one can determine the probability $p_{i j}$ that its value falls into bin $j$ of the histogram on the hadron level if it occupies bin $i$ on the parton level. The prediction from equation (1) was folded with this response matrix before fitting the data.

- The energy-energy correlation does not characterize an event by a single number, i.e. it is not a global shape variable. The response matrix formalism therefore cannot be applied. In this case the ratio of hadron and parton level distributions is determined and equation (1) is multiplied by this ratio.

In all cases the magnitude of the correction enters the theoretical error estimate. One should therefore try to find variables where the corrections are very small, i.e. typically less than $10 \%$.

For the study of systematic errors inherent in the correction based on the ME model the transition from the parton level to the hadron level was studied in addition for the following models:

- Lund ME model using $\mu^{2}=0.002 \cdot M_{Z}^{2}$ with parameters tuned to the data. The small scale used in this version of the ME model implies that the higher order effects hidden in the response matrix are significantly changed. The sensitivity of $\alpha_{t}\left(M_{Z}^{2}\right)$ to this change hence probes the importance of higher order effects.

- Lund Parton Shower (PS) model [12] as implemented in the program JETSET version 7.2. This model uses an $\mathcal{O}\left(\alpha_{0}\right)$ matrix element together with a quark-gluon shower simulated in the framework of the leading-logarithm approximation. Since there is no well-defined correspondence between a fixed order perturbative calculation and the number of branchings in the parton shower, the transition of partons into hadrons was studied for parton shower stages with different virtual parton masses of $13 \mathrm{GeV}$ and $7.3 \mathrm{GeV}$. These values were chosen such that a second order perturbative parton state should be included. At 13 $\mathrm{GeV}$ the average parton multiplicity of the shower is the same as given by the ME model with $\mu^{2}=M_{Z}^{2}$. At $7.2 \mathrm{GeV}$ the average parton multiplicity of the shower is four, the maximal value possible in an $\mathrm{ME}$ model.

The theoretical error due to higher order perturbative and non-perturbative effects is defined to be the maximum deviation of the corrected value of $\alpha_{\bullet}\left(M_{Z}^{2}\right)$ from the results using any of these alternative response matrices.

In order to separate the effects of perturbative higher order effects from the non-perturbative final hadronization process, the transition of the final shower states into hadrons was also studied in PS models. The corresponding response matrices were determined for the Lund PS and the 
HERWIG [13] models which are both based on the leading-logarithm approximation but employ very different hadronization schemes.

\section{Determination of $\alpha_{,}\left(M_{Z}^{2}\right)$ from Energy-Energy Correlation of Clusters}

A widely used non-global event shape variable in the framework of $\alpha$, measurements is the energy-energy correlation (EEC) and its asymmetry (AEEC), proposed in references [14] and studied by several experiments at lower energies [15] and at LEP [16]. The AEEC, in particular, is expected to be independent of fragmentation effects at high energies. In addition it has been found that it offers a measure of $\alpha$, which is insensitive to the renormalization scale [16].

The EEC is defined by the expression

$$
\operatorname{EEC}(\cos \chi)=\frac{1}{N} \sum_{\text {event, }} \sum_{i, j} \frac{E_{i} E_{j}}{E_{v i s}^{2}} \delta\left(\cos \chi-\cos \chi_{i j}\right),
$$

where $N$ is the number of events, the indices $i, j$ run over all particles in a given event, $E_{i}$ is the energy of the $i^{t h}$ particle, and $\chi_{i j}$ is the angle between the momenta of particles $i$ and $j$. The AEEC is defined as:

$$
\operatorname{AEEC}(\cos \chi)=\operatorname{EEC}(-\cos \chi)-\operatorname{EEC}(\cos \chi)
$$
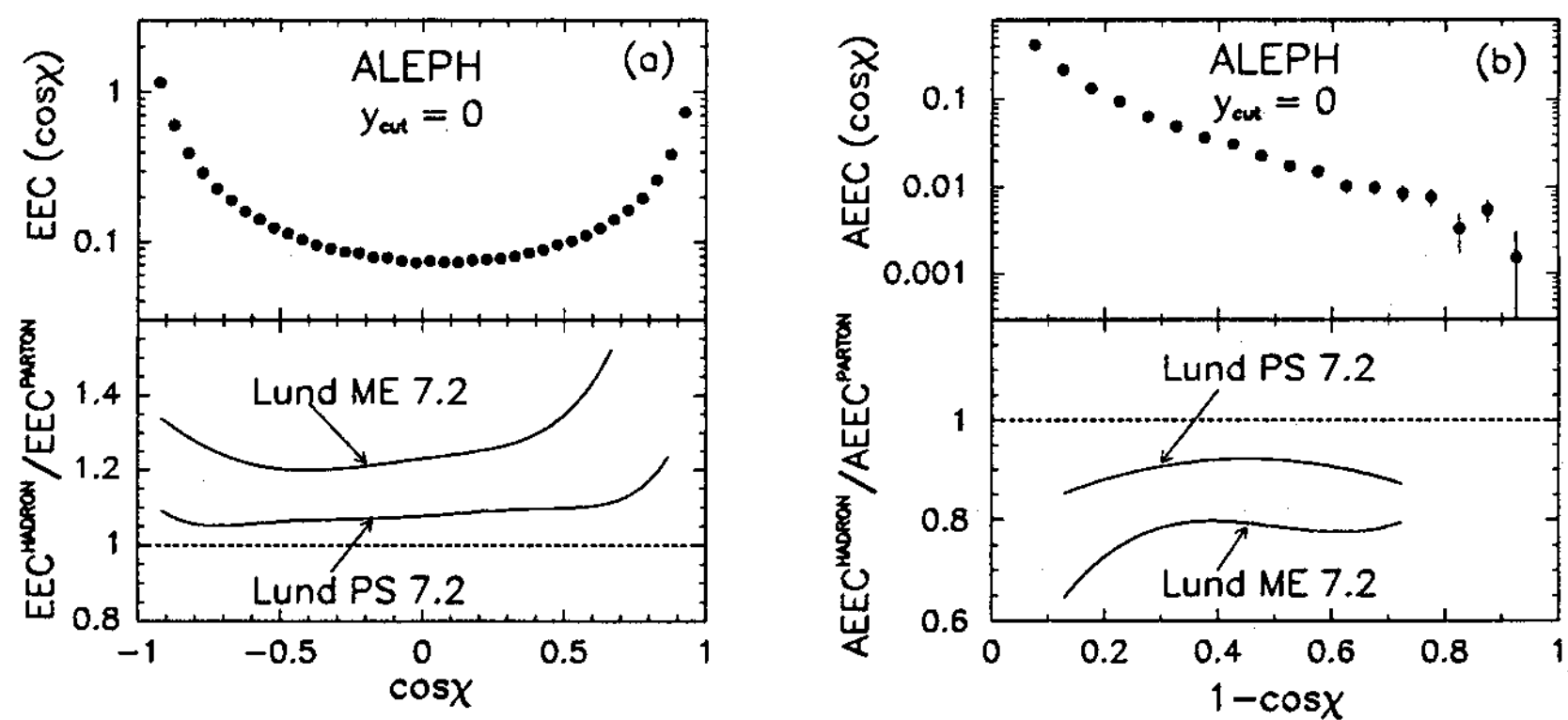

Figure 2: Measured EEC (a) and AEEC (b) distributions together with the ratio of distributions on hadron and parton level, as derived from the Lund ME model and the Lund PS model.

The experimental result for the EEC and AEEC distributions are shown in figure 2 together with the prediction of the hadron and parton level distributions from model calculations. In the Lund PS model these ratios deviate from 1 by at least $5-10 \%$, indicating a moderate sensitivity of the EEC and AEEC distributions to hadronization effects. The ratios derived from the Lund ME model, however, deviate from 1 by more than $20 \%$, suggesting that higher order perturbative corrections are very important even at LEP energies. Therefore one expects relatively large theoretical uncertainties for $\alpha$, if derived from these distributions.

The systematic distortions of the EEC distribution vanish to a large extent if one considers pre-clustered events (CEEC variable). This is shown in figure 3 where the measured CEEC 


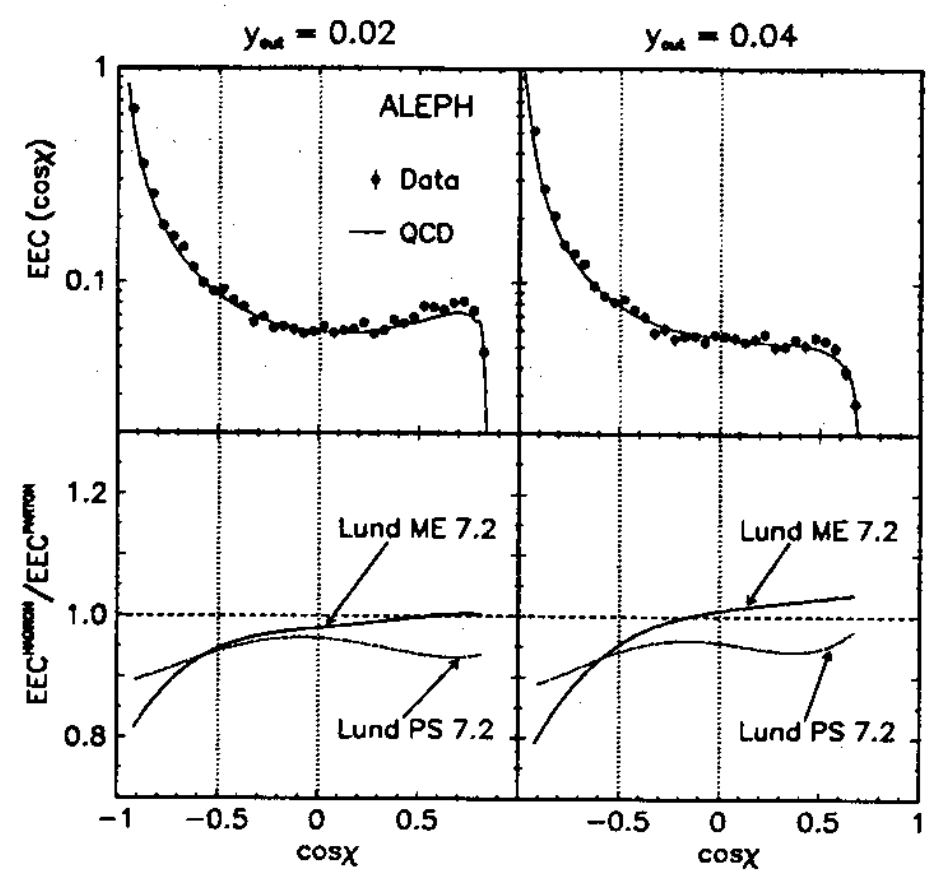

Figure 3: Measured CEEC distribution together with the ratio of CEEC distributions on hadron and parton level from model calculations for two values of $y_{\text {cut }}$. Also shown are second order $Q C D$ predictions for $\alpha_{3}\left(M_{Z}^{2}\right)=0.118$ and $\mu=M_{Z} / 2$ after correction for fragmentation effects.

distribution is displayed together with the ratio of hadron and parton level distributions from model calculations for two values of $y_{\text {cut }}$. The distortions due to higher order perturbative effects and due to hadronization are very small in the central range. The CEEC distribution changes in shape with varying $y_{\text {cut }}$. This is quantitatively predicted by the second order QCD calculation which is shown in figure 3 after correction using the Lund ME model. The curves correspond to $\alpha_{s}\left(M_{Z}^{2}\right)=0.118$ for a renormalization scale $\mu=M_{Z} / 2$.

The systematic effects for the AEEC can be also somewhat reduced by the pre-clustering but they remain larger than $15 \%$. Also, after pre-clustering the strong coupling constant derived from AEEC was found to be again more strongly dependent on the renormalization scale. The AEEC is therefore not further considered in this discussion of pre-clustered shape variables.

Although the residual fragmentation effects for the CEEC are small they are slightly asymmetric in $\cos \chi$. From the results of all models (described in the previous section), it is found that the range $-0.5<\cos \chi<0$ has consistently small and constant distortions. This range is therefore chosen to fit $\alpha_{s}\left(M_{Z}^{2}\right)$. The change of the results when moving to a symmetric range $-0.25<\cos \chi<0.25$ has been included in the theoretical error. The results from fits of the second order QCD prediction to the data are presented in figure 4 as function of $y_{\text {cut }}$ before correction for residual fragmentation effects (figure 4(a)), and after correction with theoretical uncertainties for a fixed scale $\mu=M_{Z} / 2$ included in the errors. It is obvious that for $y_{\text {cut }} \rightarrow 0$ the fits become unstable as expected from the large influence of higher order corrections. Already for $y_{\text {cut }}>0.0025$, however, this instability dies out and the result for $\alpha_{s}\left(M_{Z}^{2}\right)$ no longer depends on $y_{\text {cut }}$.

Finally $y_{\text {cut }}=0.02$ was chosen. The uncorrected value, $\alpha_{s}^{\text {uncorr }}$, the range of values corrected for higher order and hadronization effects, $\alpha_{s}^{\text {corr }}$, and the range of values corrected for 

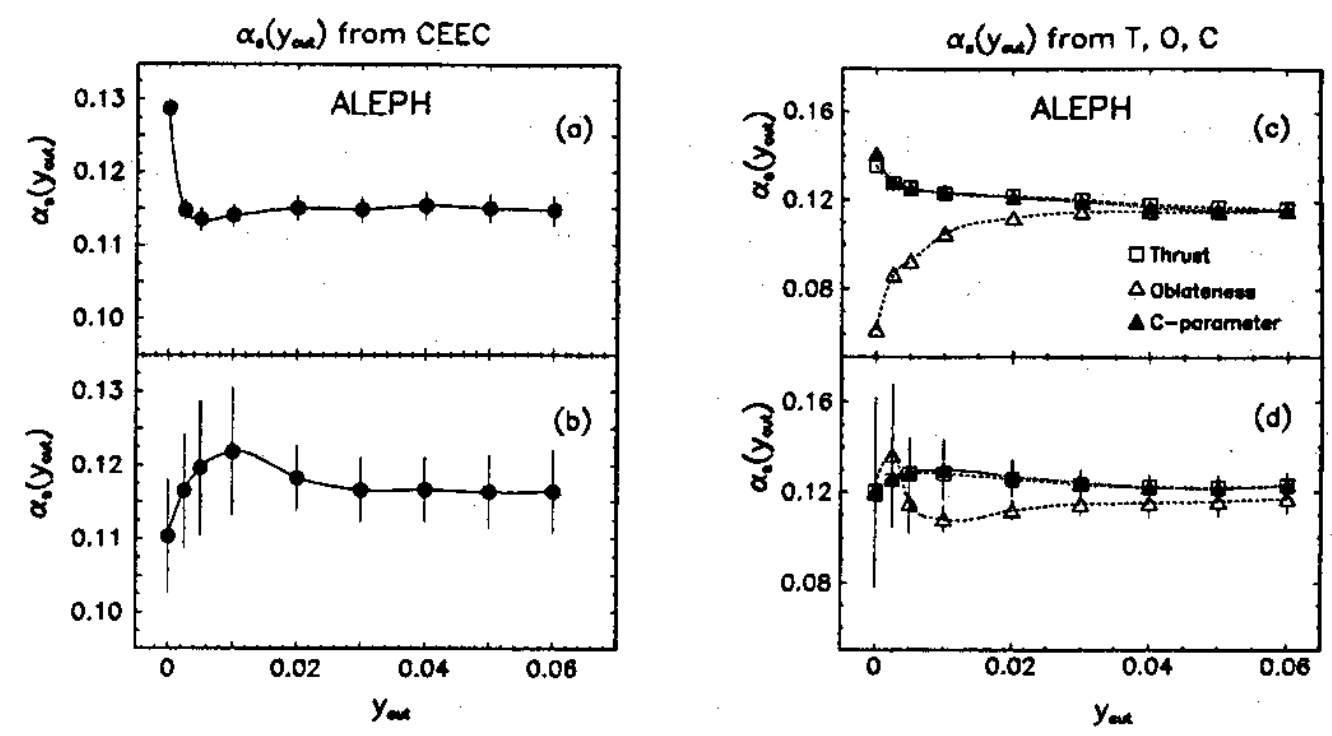

Figure 4: Results for $\alpha_{s}\left(M_{Z}^{2}\right)$ as function of $y_{\text {cut }}$ from $Q C D$ fits to event shape distributions before correction for fragmentation effects $(a, c)$, and after correction with estimated theoretical uncertainties for a fixed scale $\mu=M_{Z} / 2$ included in the errors $(b, d)$.

hadronization alone, $\alpha^{\text {had }}$, are listed in table 1 . From these one finds

$$
\left.\alpha_{\star}\left(M_{Z}^{2}\right)\right|_{\mathrm{CEEC}}=0.118 \pm 0.002 \pm 0.001 \pm 0.005 \text { for } \mu=\frac{M_{Z}}{2}
$$

the errors denoting the statistical error, the experimental systematic error, and the theoretical uncertainties defined in section 3.4. Varying $\mu$ between $M_{Z}$ and the b-quark mass changes the result in addition by ${ }_{-0.010}^{+0.006}$. The dependence of the uncorrected result on the choice of $f=\mu^{2} / M_{Z}^{2}$ was found to be

$$
\alpha_{\bullet}\left(M_{Z}^{2}, f\right)-\alpha_{\bullet}\left(M_{Z}^{2}, f=0.25\right)=0.00371 \ln (4 f)+0.00034 \ln ^{2}(4 f)
$$

for $f \geq 0.002$.

The analysis was repeated using the covariant $E$ recombination scheme [8] together with the JADE metric. The theoretical errors are only slightly larger in this case, and the corrected value for $\alpha_{s}\left(M_{Z}^{2}\right)$ agrees with the result quoted above.

\section{Influence of Clustering on Global Event Shape Variables}

The recent determination of $\alpha$, from a wide variaty of global event shape variables showed that most variables are still strongly affected by higher order effects and the hadronization process [3]. In this section the variables thrust, $T$, oblateness, $O$, and the $C$-parameter, as defined in reference [8], are reconsidered in the context of pre-clustered events.

The fits of $\alpha_{s}\left(M_{Z}^{2}\right)$ were performed in intervals contained in those chosen in reference [3]. In addition it was required that the ranges are well inside the kinematic boundaries imposed on the event shape distribution due to pre-clustering up to $y_{\text {cut }}=0.06$. The ranges are listed in table 1 . The results of the fits are displayed in figure $4(\mathrm{c}, \mathrm{d})$ as function of $y_{\text {cut }}$ before correction for fragmentation effects, and after correction with theoretical errors for a fixed renormalization scale $\mu=M_{Z} / 2$ included.

Similarly to the CEEC, the fitted $\alpha_{s}\left(M_{Z}^{2}\right)$ becomes stable for $y_{\text {cut }}>0.02$. At the same time the results from the various event shape variables are approaching each other and the theoretical uncertainties decrease. The stabilization is slower, however, than for CEEC. 


\begin{tabular}{|c|c|c|c|c|}
\hline Variable & Fit Interval & $\alpha_{o}\left(M_{Z}^{2}\right)^{\text {uncorr }}$ & $\alpha_{s}\left(M_{Z}^{2}\right)^{\text {corr }}$ & $\alpha_{s}\left(M_{Z}^{2}\right)^{\text {had }}$ \\
\hline CEEC, $y_{\text {cut }}=0.02$ & $-0.5-0.0$ & $0.115 \pm 0.002$ & $0.115-0.123$ & $0.119-0.124$ \\
T, $y_{\text {cut }}=0.03$ & $0.8-0.9$ & $0.120 \pm 0.003$ & $0.118-0.125$ & $0.126-0.128$ \\
C, $y_{\text {cut }}=0.03$ & $0.4-0.6$ & $0.119 \pm 0.003$ & $0.119-0.127$ & $0.125-0.127$ \\
$\mathrm{O}, y_{\text {cut }}=0.03$ & $0.3-0.4$ & $0.114 \pm 0.003$ & $0.113-0.120$ & $0.118-0.120$ \\
\hline
\end{tabular}

Table 1: Fit intervals, values for $\alpha_{s}\left(M_{Z}^{2}\right)$ before correction for fragmentation effects $\left(\alpha_{s}^{u n c o r r}\right)$, range of corrected values $\left(\alpha_{s}^{\text {corr }}\right)$, and range of values corrected for hadronization alone $\left(\alpha_{s}^{\text {had }}\right)$. The renormalization scale $\mu=M_{Z} / 2$ applies throughout.

In order to understand better the relevance of these observations one has to take into account the extent to which the global event shape variables become more strongly correlated due to the pre-clustering. At $y_{\text {cut }}=0.06$, for example, almost all events in the fit ranges consist of exactly three clusters. Although the $E_{0}$ scheme (defined in section 3.1) leads to slight violation of momentum conservation, these three cluster events are almost planar and their kinematic structure can therefore be described by only two independent variables. Therefore, in such an extreme case, there cannot be more than two independent event shape variables. Thrust, for example, becomes equivalent to the differential two jet rate, $y_{3}: y_{3}=1-T$.

\begin{tabular}{|c|c|c|c|}
\hline$y_{\text {cut }}$ & $\mathrm{T}, \mathrm{O}$ & $\mathrm{T}, \mathrm{C}$ & $\mathrm{O}, \mathrm{C}$ \\
\hline 0 & $-0.198 \pm 0.025$ & $-0.816 \pm 0.001$ & $0.196 \pm 0.033$ \\
& $7.5 \%$ & $68.1 \%$ & $3.8 \%$ \\
\hline 0.03 & $-0.304 \pm 0.013$ & $-0.702 \pm 0.004$ & $0.534 \pm 0.011$ \\
& $36.0 \%$ & $65.2 \%$ & $45.4 \%$ \\
\hline 0.06 & $-0.328 \pm 0.013$ & $-0.709 \pm 0.004$ & $0.529 \pm 0.010$ \\
& $39.5 \%$ & $65.9 \%$ & $50.0 \%$ \\
\hline
\end{tabular}

Table 2: Linear correlation coefficients of all pairs of global event shape variables inside the chosen fitting ranges together with the percentage of overlap of the data samples used for the two variables. All numbers are derived from data.

The correlation between the global event shape variables inside the fit intervals is quantified in table 2, where the linear correlation coefficients for any two variables inside their fitting ranges are presented. The overlaps of the samples corresponding to the two fit intervals are also listed. All the numbers are deduced from real data. It can be seen that in the case of $T$ and $\mathrm{C}$ both the correlation and the overlap are substantial, independent of $y_{\text {cut }}$. It is therefore not surprising that these two variables yield very similar values for $\alpha_{s}\left(M_{Z}^{2}\right)$. In the case of $\mathrm{T}$ and $\mathrm{O}$, on the other hand, overlap and correlation stay moderate up to $y_{\text {cut }}=0.06$. These variables are therefore contributing significant independent information and the similarity of the fit results for $\alpha_{s}\left(M_{Z}^{2}\right)$ cannot be explained by simple kinematics.

The smallest value of $y_{\text {cut }}$ at which all three variables start to give stable values for $\alpha_{s}\left(M_{Z}^{2}\right)$ is 0.03 . The measured distributions for this cut-off are displayed in figure 5 together with the QCD fits. It can be seen that the data are described for a much larger range than the fit interval. The good fit for the Oblateness distribution is especially remarkable since this variable 

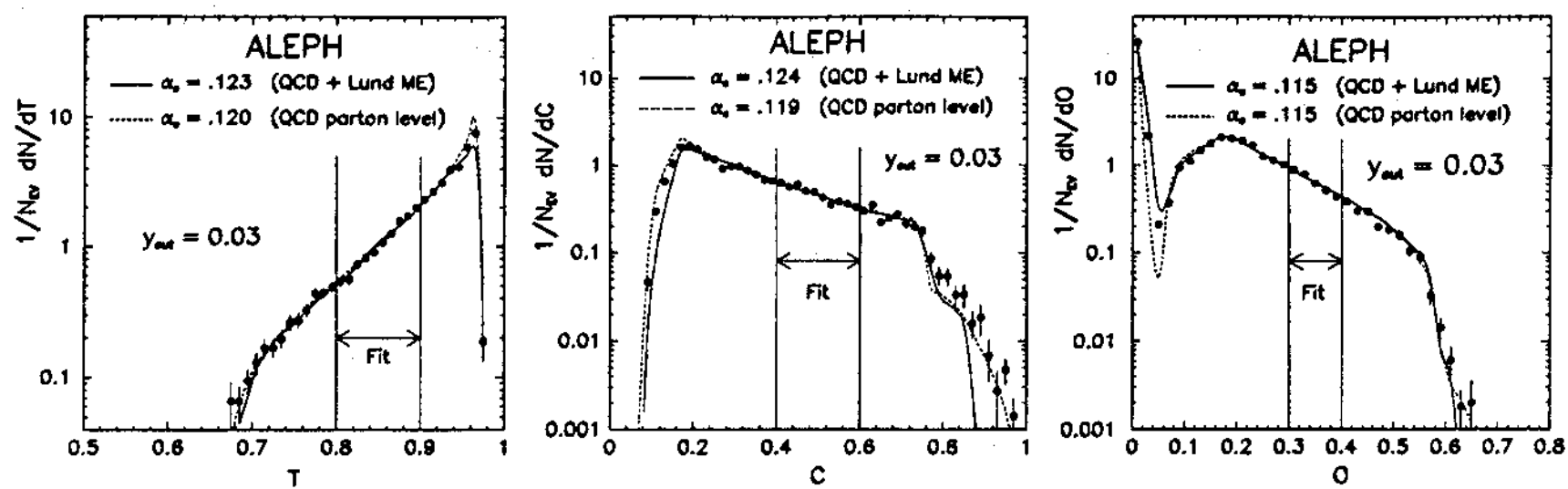

Figure 5: Measured distributions for $T, C$ and $O$ for $y_{\text {cut }}=0.03$ together with uncorrected and corrected $Q C D$ predictions for $\mu=M_{Z} / 2$.

\begin{tabular}{|l|c|c|c|c|}
\hline Distribution & $\alpha_{s}\left(M_{Z}^{2}\right), f=0.25$ & $c_{1}$ & $c_{2}$ & $f_{\min }$ \\
\hline CEEC & $0.118 \pm 0.002 \pm 0.005$ & 0.00371 & 0.00034 & 0.002 \\
$\mathrm{~T}$ & $0.123 \pm 0.004 \pm 0.006$ & 0.00449 & 0.00035 & 0.001 \\
$\mathrm{C}$ & $0.124 \pm 0.004 \pm 0.006$ & 0.00427 & 0.00037 & 0.001 \\
$\mathrm{O}$ & $0.115 \pm 0.004 \pm 0.005$ & 0.00375 & 0.00035 & 0.001 \\
\hline
\end{tabular}

Table 3: Results for $\alpha_{s}\left(M_{Z}^{2}\right)$ with combined experimental errors and errors due to model corrections together with coefficients of a parameterization for the change $\alpha_{s}(f)-\alpha_{s}(f=0.25)=c_{1} \cdot \ln (4 f)+c_{2} \cdot \ln ^{2}(4 f)$. The last column gives a lower limit for the scale parameter $f$ where the parameterization is still within the statistical error of the fitted value.

is poorly described by second order QCD if no pre-clustering is applied [3]. The ranges of fitted values for $\alpha_{s}\left(M_{Z}^{2}\right)$ leading to the estimate of uncertainties due to hadronization and higher order perturbative effects are summarized in table 1 in analogy to CEEC. The final results for $\alpha_{s}\left(M_{Z}^{2}\right)$ together with experimental errors and uncertainties due to fragmentation effects are summarized in table 3 . In addition the scale dependence was parameterized by a function

$$
\alpha_{s}\left(M_{Z}^{2}, f\right)-\alpha_{s}\left(M_{Z}^{2}, f=0.25\right)=c_{1} \ln (4 f)+c_{2} \ln ^{2}(4 f) .
$$

The parameters $c_{1}, c_{2}$ together with the lowest value of $f$ for which the parameterization stays within the statistical error of the fitted $\alpha_{\mathfrak{s}}\left(M_{Z}^{2}, f\right)$ are also listed in table 3 .

The results in table 3 are not only consistent with each other but also very close to earlier measurements using $y_{3}[1,2,3]$ and EEC or AEEC [16]. The fragmentation effects for all pre-clustered variables are small and the theoretical uncertainties are dominated by the scale dependences.

\section{Combination of the Results}

In order to derive a final result from the numbers in table 3 , the correlations between the statistical and between the theoretical errors of the variuos measurements were determined. For the statistical errors the data were divided into 21 independent sub-samples and $\alpha$, was determined from each sample for all four event shape variables. From this the full $4 \times 4$ covariance matrix was computed. Similarly, the results for the various models used for the definition of the 
theoretical uncertainty were used to construct a covariance matrix for the strongly correlated theoretical errors. The sum of the two covariance matrices was then used to calculate a weighted average. The combined result for $\mu=M_{Z} / 2$ is

$$
\alpha_{\lrcorner}\left(M_{Z}^{2}\right)=0.117 \pm 0.005,
$$

where the error contains both experimental and theoretical errors. The combined scale dependence is then

$$
\alpha_{2}\left(M_{Z}^{2}, f\right)-\alpha_{s}\left(M_{Z}^{2}, f=0.25\right)=0.00356 \ln (4 f)+0.00035 \ln ^{2}(4 f)
$$

which leads to a variation of ${ }_{-0.009}^{+0.006}$ for scales ranging from the b-quark mass up to $M_{Z}$.

\section{Conclusions}

The strong coupling constant has been measured from an analysis of the structure of preclustered events. Energy-energy correlation, Thrust, C-parameter and Oblateness all yield consistent values for $\alpha_{s}\left(M_{Z}^{2}\right)$ with moderate theoretical errors. The combined result of all four variables is $\alpha_{s}\left(M_{Z}^{2}\right)=0.117 \pm 0.005$ for $\mu=M_{Z} / 2$, where renormalization scales varying between the b-quark mass and $M_{Z}$ lead to changes of ${ }_{-0.009}^{+0.006}$. The combined value is almost identical to that obtained from CEEC alone, indicating that the results from the different event-shape variables have strongly correlated theoretical uncertainties. The final value is also in good agreement with our earlier measurement from $y_{3}, \alpha_{s}\left(M_{Z}^{2}\right)=0.121 \pm 0.002($ stat. $) \pm 0.003($ syst. $) \pm$ $0.007(\text { theory })_{-0.012}^{+0.007}($ scale $)[3]$.

\section{Acknowledgements}

We would like to thank $P$. Nason for very useful discussions and his help with the theoretical predictions for pre-clustered events. We congratulate our colleagues of the LEP division for the excellent performance of the storage ring. Thanks are also due to engineers and technical personnel at all collaborating institutions for their support in constructing and maintaining . Those of us from non-member states thank CERN for its hospitality. 


\section{References}

[1] S. Komamiya et al., MarkII Collaboration, Phys. Rev. Lett. 64 (1990) 937.

[2] M.Z. Akrawy et al., OPAL Collaboration, Phys. Lett. B235 (1990) 389;

M.Z. Akrawy et al., OPAL Collaboration, CERN-PPE/90-143;

P. Abreu et al., DELPHI Collaboration, Phys. Lett. B247 (1990) 167;

B. Adeva et al., L3 Collaboration, Phys. Lett. B248 (1990) 462.

[3] D. Decamp et al., ALEPH Collaboration, CERN-PPE/90-176.

[4] ALEPH-a Detector for Electron-Positron Annihilation at LEP, Nucl. Instr. Meth. A294 (1990) 121.

[5] D. Decamp et al., ALEPH Collaboration, Phys. Lett. B234 (1990) 209.

[6] W. Bartel et al., JADE Collaboration, Z. Phys. C33 (1986) 23;

S. Bethke et al., JADE Collaboration, Phys. Lett. B213 (1988) 235.

[7] R.K. Ellis, D.A. Ross and A.E. Terrano, Nucl. Phys. B178 (1981) 421.

[8] Z. Kunszt, P. Nason, G. Marchesini and B.R. Webber, $Q C D$, in Proceedings of the Workshop on $Z$ Physics at LEP, eds. G. Altarelli, R. Kleiss and C. Verzegnassi, CERN Report 89-08.

[9] P. Nason, private communication.

[10] P.M. Stevenson, Phys.Rev. D16 (1981) 2916;

S.J. Brodsky, G.P. Lepage and P.B. Mackenzie, Phys. Rev. D28 (1983) 228;

G. Grunberg, Phys. Lett. B95 (1980) 70.

[11] T. Sjöstrand and M. Bengtsson, Comp. Phys. Comm. 43 (1987) 367.

[12] M. Bengtsson and T. Sjöstrand, Phys. Lett. 185B (1987) 435.

[13] G. Marchesini and B.Webber, Cavendish-HEP-88/7 (1988);

G. Marchesini and B. Webber, Nucl. Phys. B310 (1988) 461;

I. Knowles, Nucl. Phys. B310 (1988) 571.

[14] C. Basham et al., Phys. Rev. Lett. 41 (1978) 1585, Phys. Rev. D19 (1979) 2018 and ibid. D24 (1981) 2382;

Yu.L. Dokshitzer, D.I. D'yakonov and S.I. Troyan, Phys. Lett. 78B(1978)290.

[15] See for example, W. de Boer, Review of QCD at LEP and SLC, CERN-PPE/90-161, and references therein.

[16] P. Abreu et al., DELPHI Collaboration, CERN-PPE/90-122;

M.Z. Akrawy et al., OPAL Collaboration, CERN-PPE/90-121;

B. Adeva et al., L3 Collaboration, L3 Preprint \#023 (1990). 